Article

\title{
On Description of Acceleration of Spinless Electrons in Law of Heat Conduction a capite ad calcem in Temperature
}

\section{Kal Renganathan Sharma}

Received: 17 August 2015; Accepted: 22 December 2015; Published: 30 December 2015 Academic Editor: Vijay Kumar Thakur

Electrical Program, San Jacinto College Central Campus, 8060 Spencer Highway, Pasadena, TX 77505, USA; jyoti_kalpika@yahoo.com; Tel.: +1-281-256-2976; Fax: +1-832-879-9410

Abstract: Acceleration effects of heat flow are included in the law of heat conduction by eliminating the acceleration term between the equation of motion for a spinless electron and the Boltzmann equipartition energy theorem differentiated with respect to time. The resulting law of heat conduction is a capite ad calcem in temperature as given in Equations (17), (19) and (20).

$$
\left(\frac{q_{z}}{k}\right)_{z}=-\left(\frac{\partial T}{\partial z}\right)-\frac{1}{v_{h}}\left(\frac{\partial T}{\partial t}\right)
$$

Evaluation of use of this equation using the entropy production term reveals that as long as the flux, $q$, and the temperature accumulation both have the same signs, the law does not violate the second law of thermodynamics. For systems that obey the first law of thermodynamics, this is the case.

$$
\sigma=\frac{q}{T^{2}}\left(\frac{q}{k}+\frac{1}{v_{h}} q\left(\frac{\partial T}{\partial t}\right)\right)
$$

In the chemical potential Stokes-Einstein formulation, when acceleration of the molecule is accounted for, a law of diffusion a capite ad calcem concentration results. In cartesian one-dimensional heat conduction in semi-infinite coordinates, the governing equation for temperature or concentration was solved for by the method of Laplace transforms. The results are in terms of the modified Bessel composite function in space and time of the first order and first kind. This is when $\tau>X$. For $X>\tau$ the solution is in terms of the Bessel composite function in space and time of the first order and first kind. The wave temperature is a decaying exponential in time when $X=\tau$. An approximate expression for dimensionless temperature was obtained by expanding the binomial series in the exponent in the Laplace domain and after neglecting fourth- and higher-order terms before inversion from the Laplace domain. The Fourier model, the damped wave model and the a capite ad calcem in temperature/concentration model solutions are compared side by side in the form of a graph. The a capite ad calcem model solution is seen to undergo the convex to concave transition sooner than the damped wave model. The results of the a capite ad calcem temperature model for distances further from the surface are closer to the Fourier model solution.

Keywords: law of heat conduction; acceleration of spinless electron; free electron theory; method of laplace transforms; bessel composite function; transient temperature; second law of thermodynamics

\section{Introduction}

Onsager [1,2] noted that Fourier's model is only an approximate description of the process of heat conduction, neglecting the time needed for the acceleration of heat flow. The manifestation of 
acceleration effects in the law of heat conduction is not well understood. This study aims to develop an alternate form of heat conduction equation that depicts the acceleration effect of heat flow without violation of the second law of thermodynamics.

Not all materials behave in the same manner. Some materials exhibit different behavior over the range of their typical application. For some applications, when the experimental observations cannot be explained using theoretical models, and for some new materials, constitutive equations may be derived from plausible microscale phenomena. For example, Newton's law of viscosity cannot be applied to systems such as tomato puree or suspensions of gas and solid. Fourier's law of heat conduction may not be the best possible relation between the heat flux and temperature gradient for systems where the transfer of heat is transient. Phenomena with short time scales or short space scales or very high heat flux or very high temperature gradient have been found not to be adequately represented by Fourier's law of heat conduction.

Fourier's law of heat conduction [3] and its analogs during (i) mass diffusion: Fick's law of mass diffusion [4]; (ii) electrical conduction: Ohm's law of electricity [5]; (iii) viscous momentum transfer: Newton's law of Viscosity [6]; (iv) convection: Netwon's law of cooling [6] are laws derived that have been used in engineering practice for nearly two centuries. These laws have been developed from empirical observations at steady state. Certain times these laws are not sufficient to predict the experimental observations. There are some reasons to seek an alternate equation to Fourier's law of heat conduction (Sharma $[7,8]$ );

(i) the microscopic theory of reversibility of Onsager [2] is violated;

(ii) it neglects the time needed for the acceleration of heat flow by free electrons (Sharma, [9]);

(iii) singularities were found in a number of important industrial applications of the transient representations of temperature, concentration and velocity [10-22];

(iv) the development of Fourier's law was from observations at steady state;

(v) over-prediction of theory to experiment has been found in a number of industrial applications (Renganathan, K., [10], Sharma, [10-22]);

(vi) Landau and Lifshitz observed the contradiction of the infinite speed of propagation of heat with Einstein's light speed barrier [23];

(vii) Fourier's law breaks down at the Casimir limit [24].

A non-Fourier heat conduction equation was postulated independently by Cattaneo [25] and Vernotte [26]. This equation was first suggested by Maxwell from considerations of kinetic theory of gases [27]. Joseph and Preziosi [28], Ozisik and Tzou [29] have reviewed the work done investigating this equation. Sharma [30-33] discussed several analytical solutions for transient temperature obtained by use of this equation along with the energy balance equation. He considered a semi-infinite medium at an initial temperature of $\mathrm{T}_{0}$ subject to a constant surface temperature boundary condition for times greater than zero. The hyperbolic partial differential equation (PDE) that forms the governing equation of heat conduction was solved by a new method called relativistic transformation of coordinates. The hyperbolic PDE is multiplied by $\mathrm{e}^{\tau / 2}$ and transformed into another PDE in wave temperature. This PDE is converted to an ordinary differential equation (ODE) by a transformation using [10] a variable that is spatio-temporally symmetric. The resulting ODE is seen to be a generalized Bessel differential equation. The solution from this approach is within $12 \%$ of the exact solution obtained by Baumeister and Hamill using the method of Laplace transforms. There are no singularities in the solution. There are four regimes to the solution: (a) the inertial regime at shorter times compared with the lag time at the considered point; (b) the regime at larger distances from the surface further from the wave front $X>\tau$ characterized by the Bessel composite function of the zeroth order and first kind and; (c) the regime at shorter distances from the surface prior to the wave front at $\tau>X$ characterized by the modified Bessel composite function of the zeroth order and first kind and; (iv) the wave front regime at $\tau=X$. Expressions for penetration length and inertial lag time are developed. The comparison between the solution from the method of relativistic transformation of coordinates and the method 
of Laplace transforms was made by use of Chebyshev polynomial approximation and numerical integration. In a similar manner, the exact solution to the hyperbolic PDE is solved by the method of relativistic transformation of coordinates for the infinite cylindrical and infinite spherical medium. For the case of heating a finite slab, the Taitel paradox problem is revisited. Taitel [34] found that when the hyperbolic PDE was solved the interior temperature in the slab was found to exceed the wall temperature of the slab. This is in violation of the second law of thermodynamics (Bai and Lavine [35], Zanchini [36]). By use of the final condition in time at steady state, the wave temperature was found to become zero at steady state. This condition, when mathematically posed as the fourth condition for the second-order PDE, leads to well-bounded solutions within the bounds of the second law of thermodynamics. For systems with large relaxation times, i.e., $\tau_{r}>\frac{\pi^{2}}{a^{2} \alpha}$, subcritical damped oscillations can be seen in the temperature. In a similar manner, the transient temperature for a finite sphere and finite cylinder are also derived. Nanoscale effects in the time domain (Sharma [19]) are as important, if not more important than nanoscale phenomena in the space domain in a number of applications. When the advances in microprocessor speed are approaching the limits of physical laws on gate width and miniaturization, there is incentive to re-examine the physical laws at a level of scrutiny never done before. In this study, the application of free electron theory to derivation of a universal law of heat conduction is revisited. Acceleration of spinless electrons is not taken into account during the derivation of the law of heat conduction. When the applied force on the spinless electron by the applied temperature difference equals the drag force, the electron has attained its steady drift velocity. This can be seen later in the derivation that Fourier's law of heat conduction will lead to. When the velocity of the electron is written in terms of heat flux, the Cattaneo and Vernotte equation results. The velocity of the electron gets eliminated in the derivation. Rather than eliminating the velocity, the acceleration of the spinless electron is expressed in terms of the accumulation of energy. This can lead to a de novo law of heat conduction. In an earlier study (Sharma, [10]), the accumulation at the surface of thermal energy transfer was shown to be causative in the need for an additional term in the law of heat conduction. The non-Fourier conduction equation that results is evaluated for use in the prediction of transient temperature in a finite slab.

\section{Materials and Methods}

\subsection{Free Electron Theory}

Ohm's law of electric conduction and Fourier's law of heat conduction can be derived from the free electron theory. The range of electrical resistivity of materials varies by 30 orders of magnitude. The range of thermal conductivity of materials varies by five orders of magnitude. No one theory can predict the thermal and electrical conductivity of materials. The free electron model is used. The outermost electrons of the atom are assumed to take part in the conduction process. These electrons are not bound to the atom but are free to move through the entire solid. These electrons have been called the free electron cloud, free electron gas, or Fermi gas. A potential field due to the ion cores is assumed to be uniform throughout the solid. The free electrons possess the same free energy everywhere in the solid. Because of the electrostatic attraction between a free electron and the ion core, this potential energy will be a finite negative value.

Only energy differences are important and the constant potential can be taken to be zero. Then the only energy that has to be considered is the kinetic energy. The kinetic energy is substantially lower than that of the bound electrons in an isolated atom as the field of motion for the free electron is considerably enlarged in the solid as compared to the field around an isolated atom. The free electron theory can be used to better understand electrical conduction.

By the Lorenz analogy [37], the heat conduction can also be predicted in a similar manner. The independent electron assumption was developed by Drude [38]. Some of the assumptions in the free electron theory claim that electrons are responsible for all of the conduction. The electrons behave 
like an ideal gas, occupy negligible volume, undergo collisions, and are perfectly elastic. Electrons are free to move in a constrained flat bottom well. The electron distribution of energy is a continuum.

\subsection{Derivation of Alternate Non-Fourier Conduction Equation}

Let the number of electrons per unit volume be given by $n$. This is the electron density in the material during the conduction process. From the Boltzmann equipartition energy theorem [39] the microscopic temperature, $T$, in $\left({ }^{\circ} \mathrm{K}\right)$ can be given by;

$$
\left(\frac{3 k_{B} T}{2}\right)=\left(\frac{m v_{e}^{2}}{2}\right)
$$

where $\mathrm{k}_{\mathrm{B}}$ is the Boltzmann constant, $\mathrm{m}$ is the mass of the electron in $(\mathrm{kg})$ and $v_{\mathrm{e}}$ is the velocity of the free electron in $\left(\mathrm{m} \mathrm{s}^{-1}\right)$. The heat flux, $q_{\mathrm{z}}$, in $\left(\mathrm{W} \mathrm{m}^{-2}\right)$ is the rate of energy transfer across a cross-sectional area of the heat conduction path. The heat flux in terms of the properties of the moving electron can be written as follows:

$$
q_{z}=-\left(\frac{3 k_{B} T n v_{e}}{2}\right)=\left(\frac{m n v_{e}^{3}}{2}\right)
$$

Equation (2) is the product of Equation (1) and (nve). Currently, the rate of energy transfer across surfaces is estimated this way. This is sort of the momentum flux of the electrons as well. The applied temperature gradient acts as a driving force for the motion of the electron. Let the collision time of the electron be given by $\tau$ in units of seconds. The applied force and frictional force can be written from the free electron theory as follows:

$$
\begin{gathered}
\text { Applied Force }=-\frac{3 k_{B}}{2}\left(\frac{d T}{d z}\right) \\
\text { Drag Force }=\left(\frac{m v_{e}}{\tau}\right)
\end{gathered}
$$

An earlier study, the acceleration of the spinless electron, is also taken into account. The derivations in the literature for Fourier's law of heat conduction from free electron theory assume that the electron has attained a steady drift velocity. This assumption may be reasonable at steady state. However, in transient applications, immediately after the application of the temperature gradient, the electron would be in acceleration. This phase of motion of the electron has not been well studied in the literature. In this study, this is taken into account. Newton's second law of motion may be applied to the moving electron(s) as follows:

$$
m\left[\frac{d v_{e}}{d t}\right]=-\left(\frac{3 k_{B}}{2}\right)\left(\frac{d T}{d z}\right)-\left(\frac{m v_{e}}{\tau}\right)
$$

Eliminating the velocity of the electron in Equation (5) by using Equation (2) and taking the derivative of the velocity of electron as the time derivative of heat flux leads to the damped wave conduction and relaxation equation as shown earlier (Sharma [40,41]). By the equi-partitition energy theorem given in Equation (1), the velocity of the electron can be written as follows:

$$
v_{e}=\sqrt{\frac{3 k_{B} T}{m}}
$$

Differentiating Equation (6) with respect to time, and multiplying with the mass of the electron, $m$, Equation (6) can be seen to be:

$$
\sqrt{\frac{3 m k_{B}}{4 T}}\left(\frac{\partial T}{\partial t}\right)=m\left(\frac{\partial v_{e}}{\partial t}\right)
$$


Eliminating the acceleration of the electron term between Equations (5) and (7) and plugging Equation (2) for heat flux in place of the velocity of the electron:

$$
\sqrt{\frac{3 m k_{B}}{4 T}}\left[\frac{\partial T}{\partial t}\right]=-\left(\frac{3 k_{B}}{2}\right)\left(\frac{d T}{d z}\right)-\left(\frac{2 m q_{z}}{3 k_{B} n T \tau}\right)
$$

Multiplying Equation (8) by $\left(\frac{3 k_{B} T \tau}{2 m}\right)$ :

$$
\sqrt{\frac{27 m k_{B}^{3} n^{2} T^{2} \tau^{2}}{16 m^{2} T}}\left[\frac{\partial T}{\partial t}\right]=-\left(\frac{9 k_{B}^{2} n T \tau}{4 m}\right)\left(\frac{d T}{d z}\right)-q_{z}
$$

At steady state:

$$
q_{z}=-\left(\frac{9 k_{B}^{2} n T \tau}{4 m}\right)\left(\frac{d T}{d z}\right)
$$

Equation (10) is Fourier's law of heat conduction. The expression for thermal conductivity of the material from Equation (10) can be seen to be:

$$
k=\left(\frac{9 k_{B}^{2} n T \tau}{4 m}\right)
$$

The coefficient to the rate of temperature variation with the time term in Equation (9) can be simplified as follows. The rate of temperature variation with time can be called the ballistic term. This term is unique to the acceleration of the electron and is expected to become significant in transient applications. The term "ballistic" can be used to denote acceleration effects. In an earlier study (Sharma [41]), the collision time of the electron was shown to be $3 \tau_{\mathrm{r}}$ where $\tau_{\mathrm{r}}$ is the relaxation time of the materials as defined by Cattaneo [25] and Vernotte [26].

Substituting Equation (11) in Equation (9), Equation (9) becomes:

$$
\sqrt{\frac{3 k n k_{B} \tau}{4}}\left(\frac{k \sqrt{m}}{\sqrt{3 k_{B} T}}\right)\left[\frac{\partial T}{\partial t}\right]=-k\left(\frac{d T}{d z}\right)-q_{z}
$$

In terms of properties of moving electrons, Equation (12) can be rewritten as follows. Heat capacity at constant volume, $C_{\mathrm{V}}$, by definition is the energy needed to raise one mole of a substance by $1^{\circ} \mathrm{K}$. For one molecule this would be $\left(1.5 \mathrm{k}_{\mathrm{B}}\right)$ as a corollary of the equi-partition energy theorem (Equation (1)). The electron cloud is assumed to be ideal gas. It can be shown that for an ideal gas (Sharma [31]):

$$
C_{p}=C_{v}+R
$$

Equation (13) is for one mole of the material. For one molecule of the material, Equation (13) can be written as follows:

$$
\begin{gathered}
C_{p}=C_{v}+k_{B}=2.5 k_{B} \\
\rho C_{p}=(m n)\left(\frac{5 k_{B} n}{2 m n}\right)=\frac{5 n k_{B}}{2}
\end{gathered}
$$

Plugging Equation (15) into Equation (12), Equation (12) becomes:

$$
\sqrt{\frac{3 k \rho C_{p} \tau}{10}}\left[\frac{\partial T}{\partial t}\right]=-k\left(\frac{d T}{d z}\right)-q_{z}
$$


It can be realized from Equation (16) that when the relaxation time of the material of heat propagation $\tau_{\mathrm{r}}$ is $0.3 \tau$, where $\tau$ is the collision time of the electron, Equation (16) becomes:

$$
q_{z}=-k\left(\frac{\partial T}{\partial z}\right)-\left(\rho C_{p}\right) \sqrt{\alpha \tau_{r}}\left(\frac{\partial T}{\partial t}\right)
$$

Sharma [41] showed that the relaxation time was found to be a third of the collision time of the electron with the obstacle when the Cattaneo-Vernotte-Maxwell equation of heat conduction was derived from free electron theory including the acceleration effects. Here the relaxation time is of one-third of the collision time of the electron and obstacle.

Further, it can be seen that the velocity of the electron, $v_{\mathrm{e}}$, is approximately equal to the $95 \%$ of the velocity of heat, $v_{\mathrm{h}}$. The velocity of heat is given in terms of the thermophysical properties of materials as given in Equation (17). The coefficient to the ballistic term in Equation (9) may be simplified as:

$$
\left(\frac{9 k_{B}^{2} n T \tau}{4 m v_{e}}\right)=\left(\frac{k}{v_{e}}\right)=\sqrt{\rho C_{p} k \tau_{r}}=\left(\rho C_{p}\right) \sqrt{\alpha \tau_{r}}
$$

Plugging Equation (18) into Equation (9), Equation (9) becomes:

$$
q_{z}=-k\left(\frac{\partial T}{\partial z}\right)-\left(\rho C_{p}\right) \sqrt{\alpha \tau_{r}}\left(\frac{\partial T}{\partial t}\right)
$$

Or

$$
\left(\frac{q_{z}}{k}\right)_{z}=-\left(\frac{\partial T}{\partial z}\right)-\frac{1}{v_{h}}\left(\frac{\partial T}{\partial t}\right)
$$

Equations (18)-(20) comprise an alternate non-Fourier heat conduction equation compared with that postulated by Cattaneo [25] and Vernotte [26] and Maxwell [27]. Equation (19) for heat flux comprises two parts: (i) one part is the "Fourier part" which gives the spatial gradient of the temperature, both transient and steady states; and (ii) the second part is the "ballistic part" which gives the contribution of the acceleration motion effects of the electron. In the asymptotic limit of the infinite speed of heat, $v_{\mathrm{h}}$, Equation (20) reverts to Fourier's law of heat conduction. In the asymptotic limit of the null transfer of heat, Equation (20) indicates a jump in temperature after an elapsed time at a considered point in the medium. This is at zero drag. This may not be achievable in practical applications.

Another way of viewing the "ballistic term" is in terms of the accumulation effects in time of the energy at the interfacial area through which the heat is traveling. In the kinetic representation of temperature in terms of the square of the velocity of the molecules as given by Equation (1), the heat flux across an interfacial area is defined as the energy of the molecules leaving the surface less the energy of the molecules that enter the surface per unit time. Accumulation of energy during said time at the interfacial surface was neglected in the Fourier representation of heat flux with the spatial temperature gradient in Sharma [10]. Here the accumulation of energy at the surface is also taken into account in the heat flux expression. This added term can also be viewed as a place to capture the acceleration effects of the moving electron under an applied temperature gradient/force. The postulated non-Fourier equation of Cattaneo [25] and Vernotte [26] and Maxwell [27] is restated as:

$$
q_{z}=-k\left(\frac{\partial T}{\partial z}\right)-\tau_{r}\left(\frac{\partial q_{z}}{\partial t}\right)
$$




\subsection{Entropy Production Term}

Jou [1] wrote the entropy production term in terms of the entropic flux and accumulation as follows:

$$
\sigma=\nabla\left(\frac{q}{T}\right)+\rho \frac{\partial S}{\partial t}=-\frac{\nabla T q}{T^{2}}+\frac{\nabla q}{T}+\rho\left(\frac{\partial S}{\partial t}\right)
$$

The combined statement of the first and second law of thermodynamics at constant pressure can be written as follows:

$$
d H=T d S=C_{p} d T
$$

Or

$$
T\left(\frac{\partial S}{\partial t}\right)=C_{p} \frac{\partial T}{\partial t}=-\nabla q
$$

Applying Equation (24) to Equation (22), the entropy production term becomes:

$$
\sigma=-\frac{\nabla T q}{T^{2}}
$$

For Fourier's law of heat conduction, the entropy production can be seen to be always positive and in obeyance of the second law of thermodynamics. Equation (23) can be written as follows:

$$
\left(\frac{q_{z}}{k}\right)=-\left(\frac{\partial T}{\partial z}\right)-\left(\frac{1}{v_{h}}\right)\left(\frac{\partial T}{\partial t}\right)
$$

where the finite speed of heat $v_{\mathrm{h}}$ can be seen as:

$$
v_{h}=\sqrt{\frac{\alpha}{\tau_{r}}}
$$

Eliminating $\nabla \mathrm{T}$ between Equation (26) and (25), the entropy production can be seen to be:

$$
\sigma=\frac{q_{z}}{T^{2}}\left(\frac{q_{z}}{k}+\frac{1}{v_{h}} q_{z}\left(\frac{\partial T}{\partial t}\right)\right)
$$

The entropy production can be seen to be positive for real heat events. The first term in right hand side (RHS) is always positive. The second term can give rise to a negative contribution only when the heat flux, $q$, and temperature accumulation $\left(\frac{\partial T}{\partial t}\right)$ have opposite signs. This would be a violation of an alternate statement of the second law of thermodynamics.

When the heat flux locally is positive, the accumulation in temperature at that point in that medium is also positive, i.e., the temperature increases with time at that point. It means that the heat is received at that point. Heat flux cannot be negative locally as well as the temperature rises locally at the same time. During cooling, the heat flux is negative locally and the temperature accumulation term is negative. In this case, heat is given out. The heat flux is negative as heat is taken away from that point. The temperature accumulation is also negative.

Polymer liquids are solutions of polymers and polymer in molten form. They behave in a different manner compared with the Newtonian fluids (Bird et al. [42]). The viscosity is no longer independent of the velocity gradient, causing the momentum transfer and flow of fluid when subjected to sufficient shear force. Polymers are macromolecules with higher molecular weight compared to small molecules. Staudinger defined when a molecule can be considered a macromolecule. These molecules are now believed to have many internal degrees of freedom. Pronounced "elastic effects" have been seen when practitioners worked with these fluids. Normal stresses such as $\tau_{x x}$ are needed in addition to the applied wall shear stress, $\tau_{x y}$, in order to completely describe and characterize the flow of these fluids. The viscosity changes exponentially with change in the volume fraction of the polymer in the solution. The change of viscosity with temperature is exponential as well. Master curves have been 
developed by industrial technologists and rheologists and used in the industry. Rheology is the study of deformation and flow of non-Hookean solids and non-Newtonian liquids. The Newton Law of Viscosity can be written as follows:

$$
\tau_{x y}=-\mu\left(\frac{\partial v_{y}}{\partial x}\right)
$$

where $\tau_{\mathrm{xy}}$ is the tangential shear stress and $\mu$ is the viscosity of the liquid and $v_{\mathrm{y}}$ is the velocity of the fluid in the $y$ direction. The gradient of the velocity is in the $x$ direction.

A flat plate atop a stationary fluid when pulled with a constant force will result in flow of fluid along the direction of the plate. After steady state is reached, the velocity profile in the fluid can be seen to be steady. The layers of fluid close to the plate reach speeds close to the speed of the plate. The fluid further away from the plate is unaffected and there is no motion of the fluid. The shear stress resulting from the tangential force causes the momentum direction in the azimuthal $x$ direction. The momentum transfer is in the direction of the azimuthal $x$ direction. The relation between the shear stress and shear rate is linear at steady state and is given by Equation (29). Before attaining steady state, the momentum transfer is not Newtonian. Other models such as the Maxwell model and Jeffrey model have been reported in the literature. The Maxwell viscoelastic model (Sharma [10]) can be written as:

$$
\tau_{x y}=-\mu\left(\frac{\partial v_{y}}{\partial x}\right)-\tau_{m o m}\left(\frac{\partial \tau_{x y}}{\partial t}\right)
$$

The term $\tau_{\text {mom }}$ is the relaxation time of the momentum. Sharma [9] has shown in analogous heat transfer problems that this parameter is a measure of the acceleration time of the free electron that can be used to describe Fourier conduction before it reaches steady state. It was found to be about one-third of the collision time of the free electron and an obstacle. Equation (30) has been used to characterize viscoelastic fluids where $\mu$ is the zero shear viscosity. The effects of Equation (30) exist for all transient flows, Newtonian or otherwise. However, these effects are only seen in a pronounced manner in some materials. For instance, "silly putty" is considered viscoelastic. The material flows readily when squeezed slowly using the palms of one's hands and may be considered to be in the viscous Newtonian state. The material can be rolled into a ball and the ball will rebound when dropped onto a hard surface. It can be expected that the stresses change rapidly and the material can be seen to behave analogous to an elastic solid. In some cases, Equation (30) is simplified to include only the shear rate term and the accumulation of the momentum term. This may be applicable when the changes are rapid. Sharma [9] discussed how poor use of the initial conditions can result in model solutions that may be in dissonance with the second law of thermodynamics using Equation (30). Damped wave transport and relaxation were studied. The results from an in-depth study of the Cattaneo and Vernotte non-Fourier heat conduction answered a few questions. One issue is that the entropy production becomes negative when the momentum flux is high and the momentum rate is in the opposite direction. This can lead to a violation of the second law of thermodynamics locally. The "overshoot" phenomena were shown to be a mathematical artifact and, when physically reasonable, the final time condition or the higher accumulation of the temperature condition is used and the overshoot was found to disappear. An equation to describe real mass diffusion is derived from Gibbs' chemical potential formulation for a non-relativistic solute particle. The acceleration term eliminated between the equation of motion for the spinless particle and the accumulation of chemical potential formulation leads to an equation for mass diffusion that is a capite ad calcem in concentration (Equation (39)). This entropy production for this term can be seen to be positive for real mass transfer events. The $\mathrm{d} C / \mathrm{d} t$, the time derivative of concentration and mass flux, can either be "both positive" and "both negative" but never one positive and one negative for spontaneous mass diffusion events. So for all extemporaneous and practical purposes, the second law of thermodynamics is obeyed.

The Nobel Prize in 1991 was obtained by P. G. de Gennes. He discussed in his Nobel lecture what are called "complex fluids". Natural rubber, enzymes, hydrophobic/hydrophilic surfactants, nematic crystals, ferroelectric smectics, and Ferro fluids made of magnetic particles are examples of complex 
fluids. He looked at the trajectories of non-relativistic particles and applied the Schrodinger wave equation to explain Raleigh Bernard instability. The behavior changes completely upon "cross-link" formation in the rubber. The relaxation time can be expected to be high for the cross-linked systems. When the rubber chains are elongated or present in chain-extended configuration, the relaxation time can be expected to be short.

Chrysler has introduced electrorheological fluids in the automatic transmission systems. The viscosity of the fluid changes by an order of magnitude upon application of an electrical field. The Fahreus Lindquist effect has been seen during blood flow through narrow capillaries. The viscosity of the fluid appeared to change with the diameter of the capillary. Some of these experimental observations can be explained using the viscoelastic nature of transient flow.

The Jeffrey model (Sharma, [43]) can be seen to be:

$$
\tau_{x y}=-\mu\left(\frac{\partial v_{y}}{\partial x}\right)-\tau_{m o m}\left(\frac{\partial \tau_{x y}}{\partial t}\right)-\tau_{m o m} \lambda_{2}\left(\frac{\partial^{2} v_{y}}{\partial x \partial t}\right)
$$

where $\lambda_{2}$ can is a retardation time.

Polymeric liquids were seen (Bird et al. [42]) to exhibit constrained recoil after the cessation of flow in a circular fluid in contrast to Newtonian fluid. The polymeric liquid was supposed to have "fading memory". The Weissenberg rod-climbing effect is when the polymer liquid forms a convex meniscus or moves up a rotating rod in a beaker of fluid. Au contraire, the Newtonian fluid forms a concave meniscus and forms a trough around the rotating rod in the beaker of fluid. Secondary flows have been observed in polymeric fluids when a disk is rotated in the fluid. The Weissenberg rod-climbing effect can be used in a tubeless siphon when the polymeric fluid is extruded. Near a laterally oscillating rod, the induced secondary flows in polymeric fluid are in the opposite direction of the induced secondary flow in Newtonian fluid.

\subsection{Transport Parameters}

The motion of oligonucleotide fragments in different gels is a vast subject. There are different regimes of transport where some considerations are more important than other considerations. Calibration is used in the inference of sizes in chromatographic and electrophoretic migrations. Diffusion of oligonucleotides is a phenomenon that is not well characterized under transient conditions. The critical events are the migration of molecular fragments over varying distances depending on their molecular sizes. Calibration is used in order to convert the raw measurements to sequence information. It is not clear why mathematical models are not used for interpretation of the electrophoretic pattern or the paper chromatographic pattern. It is being realized increasingly among investigators that at short time scales, Fick's description of transient diffusion is not an adequate representation of all the events. Molecular diffusion principles are revisited with particular attention to the derivation of Fick's laws of diffusion from chemical potential formulation. A generalized Fick's law of diffusion is used to account for all the transient time events that occur during a real process. The implications on the electrophoretic techniques and sequence errors and shotgun sequencing may be important. The mathematical framework to describe molecular diffusion needs to accommodate terms for the acceleration of the solute in motion. The Newtonian acceleration of a spinless solute without the Brownian dynamics, when accounted for, can lead to spatio-temporal concentration profiles that can be drawn without violating the second law of thermodynamics. Sharma [8] has listed seven reasons from the works of Nobel laureates Onsager, Landau, Nernst for the non-universality of Fick's law of diffusion. Fick's law of diffusion can be written as follows:

$$
J_{A}=-D_{A B} A\left(\frac{\partial C_{A}}{\partial z}\right)
$$


where $J$ is the molar flux with units of $\left(\right.$ mole $\left.\mathrm{m}^{-2}\right), D_{\mathrm{AB}}$ is the binary diffusivity, $C_{\mathrm{A}}$ is the concentration of the diffusing solute and $z$ is the spatial direction of the solute transfer. The solute transfer is considered to be one-dimensional. When motion in other dimensions becomes important, terms can be added for each ordinate. The diffusion coefficient $D_{\mathrm{AB}}$ is obtained from the Stokes-Einstein formulation as follows:

$$
D_{A B}=\left(\frac{k_{B} T}{f}\right)=\left(\frac{k_{B} T}{6 \pi \mu R_{0}}\right)
$$

where $\mathrm{k}_{\mathrm{B}}$ is the Boltzmann constant with units of $\mathrm{J}$ molec ${ }^{-1} \mathrm{~K}^{-1}, \mathrm{~T}$ is the absolute temperature, $f$ is the molecular drag coefficient, $\mu$ is the viscosity of the surrounding medium, and $R_{0}$ is the radius of the solute molecule. Rigid spheres are assumed. Equations (32) and (33) are derived as follows. The chemical potential of an ideal solution of solute A in solvent $B$ can be written as:

$$
\mu_{A}=\mu_{A}^{0}+k_{B} T \ln \left(x_{A}\right)
$$

The mole fraction $x_{\mathrm{A}}$ can be approximated for dilute solutions as $\left(\frac{C_{A}}{C_{B}}\right)$. At steady state, when the solute is in motion caused by the chemical potential gradient, the driving force and drag forces will be equal to each other and:

$$
f v_{A}=-\nabla \mu_{A}=k_{B} T\left(\frac{\nabla C_{A}}{C_{A}}\right)
$$

The molar flux $J^{\prime \prime}=\left(\frac{J}{A}\right)$ is given by $C_{\mathrm{A}} v_{\mathrm{A}}$ and:

$$
J_{A}^{\prime \prime}=-\left(\frac{k_{B} T}{6 \pi \mu R_{0}}\right)\left(\frac{\partial C_{A}}{\partial z}\right)
$$

The Stokes drag is $f=6 \mu \pi R_{0}$. The diffusion coefficient recovered from Equation (36) by comparing Equation (36) and Equation (32) can be seen to be the same as given for the diffusion coefficient in Equation (33). Equation (36) is at steady state. Often times, during the electrophoretic measurements there exists a time period between the start of the experiment to the time when the fragment motion can be considered to be at steady state. During this transient regime the solute molecules can be expected to undergo translational acceleration. The Newtonian acceleration effects are not accounted for in Fick's law of diffusion. The use of the Cattaneo and Vernotte equation in order to account for transient diffusion effects was discussed in Sharma [41]. The acceleration motion of the diffusing solute may be modeled by looking at the accumulation of the chemical potential. Thus, Equation (35) can be written including the accelerating term and:

$$
\left(\frac{1}{v_{m}}\right)\left(\frac{\partial \mu}{\partial t}\right)=-\nabla \mu_{A}-f v_{A}
$$

After realizing that the velocity of molecule $v_{\mathrm{m}}$ can be taken as the velocity of mass $v_{\text {mass }}$, (Sharma [34]) it is characterized by a relaxation time $\tau_{\mathrm{mr}}$ such that:

$$
v_{m}=v_{m a s s}=\sqrt{\frac{D_{A B}}{\tau_{m r}}}
$$

Equation (37) can be seen to be:

$$
J_{A}^{\prime \prime}=-D_{A B}\left(\frac{\partial C_{A}}{\partial z}\right)-\sqrt{D_{A B} \tau_{m r}}\left(\frac{\partial C_{A}}{\partial t}\right)
$$


where $\tau_{\mathrm{mr}}$ is the mass relaxation time. It is a characteristic measure of the acceleration time of the solute from the instant of application of the driving force which causes the flow to the steady state regime. It can be seen to be:

$$
\tau_{m r}=\left(\frac{D_{A B} N}{R T}\right)
$$

where $N$ is the molecular weight of the oligonucleotide. The velocity of mass from the Maxwell speed distribution of molecules for ideal gases can be written as in Sharma [44]:

$$
v_{\text {mass }}=\sqrt{\frac{D_{A B}}{\tau_{m r}}}
$$

\section{Results and Discussion}

Consider a semi-infinite medium with homogenous density and binary diffusivity and mass relaxation time properties as shown in Figure 1.

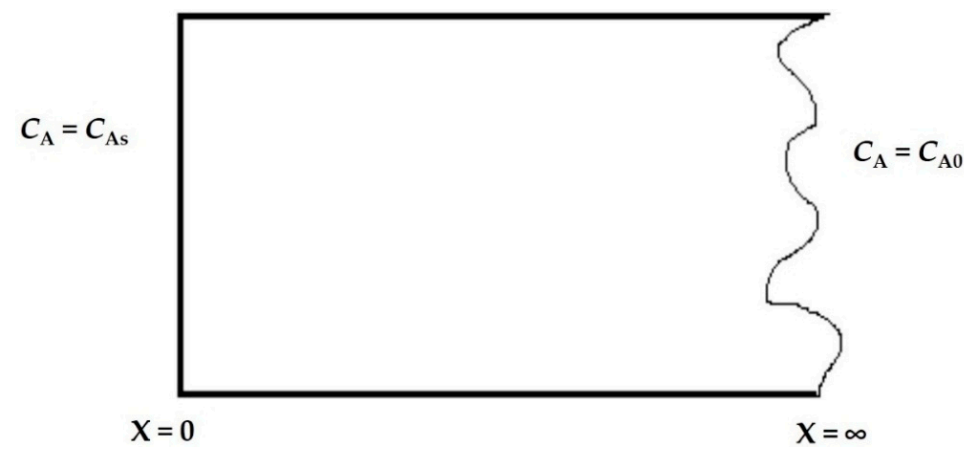

Figure 1. Semi-infinite medium subject to constant wall concentration boundary condition.

The initial concentration of the solute diffusing in the semi-infinite medium is $C_{0}$ for times less than 0 . At time 0 the surface of the semi-infinite medium is raised to a solute concentration $C_{\mathrm{As}}$ $\left(C_{\mathrm{As}}>C_{0}\right)$ and maintained constant at $C_{\mathrm{s}}$ for all times $t>0$. The initial time condition and the boundary conditions can be written as follows:

$$
\begin{gathered}
t=0,0<x<\infty, C_{\mathrm{A}}=C_{0} \\
t>0, x=0, C_{\mathrm{A}}=C_{\mathrm{As}} \\
t>0, x=\infty, C_{\mathrm{A}}=C_{0}
\end{gathered}
$$

The mass balance equation in one dimension (1D) can be written as follows:

$$
-\left(\frac{\partial J_{x}^{\prime \prime}}{\partial x}\right)=\left(\frac{\partial C_{A}}{\partial t}\right)
$$

The governing equation for solute concentration $C_{\mathrm{A}}$ in one dimension is obtained by combining Equations (39) and (45) and can be written as follows:

$$
\left(\frac{\partial C_{A}}{\partial t}\right)=D_{A B}\left(\frac{\partial^{2} C_{A}}{\partial x^{2}}\right)+\left(\sqrt{D_{A B} \tau_{m r}}\right)\left(\frac{\partial^{2} C_{A}}{\partial x \partial t}\right)
$$

Equation (46) may be made dimensionless by the following substitutions:

$$
Z=\left(\frac{x}{\sqrt{D_{A B} \tau_{m r}}}\right) ; \tau=\left(\frac{t}{\tau_{r}}\right) ; u=\left(\frac{C_{A}-C_{0}}{C_{A s}-C_{0}}\right)
$$


Equation (46) becomes:

$$
\left(\frac{\partial u}{\partial \tau}\right)=\left(\frac{\partial^{2} u}{\partial Z^{2}}\right)+\left(\frac{\partial^{2} u}{\partial Z \partial \tau}\right)
$$

The dimensionless distance $Z$ can be rewritten as:

$$
Z=\frac{\frac{x}{\tau_{n r}}}{v_{\text {mass }}=\sqrt{\frac{D_{A B}}{\tau_{m r}}}}
$$

The physical significance of the dimensionless distance can be seen to be the ratio of the relaxation speed calculated for the disturbance to be seen at the considered point to the speed of the composite mass transfer in the medium due to both Fick diffusive and ballistic/relaxation/acceleration mechanisms. The approximate solution to Equation (46) may be obtained by the method of Laplace transforms (Sharma [30]). The Laplace transformed Equation (46) may be written as follows:

$$
s u(s)=\frac{d^{2} u(s)}{d Z^{2}}+s \frac{d u(s)}{d Z}
$$

The solution to the second-order ODE given by Equation (50) can be written as follows:

$$
u(s)=e^{-\frac{s Z}{2}}\left(c_{1} e^{\frac{s Z}{2} \sqrt{s(s+4)}}+c_{2} e^{-\frac{s Z}{2} \sqrt{s(s+4)}}\right)
$$

From the undisturbed temperature, at ad infinitum, as given by boundary condition (BC), in Equation (44), $c_{1}$ can be seen to be zero.

$$
u(s)=e^{-\frac{s Z}{2}}\left(c_{1} e^{\frac{s Z}{2} \sqrt{s(s+4)}}+c_{2} e^{-\frac{s Z}{2} \sqrt{s(s+4)}}\right)
$$

The term $c_{2}$ is obtained from the constant wall temperature BC as given in Equation (43) and is seen to be given by $(1 / \mathrm{s})$. The solution for the dimensionless temperature in the Laplace domain may be written as follows:

$$
u(s)=\frac{1}{s} e^{-\frac{s Z}{2}}\left(e^{-\frac{Z}{2} \sqrt{s(s+4)}}\right)
$$

The lag property in the Laplace transforms is invoked as follows Equation (50):

$$
F(s)=\frac{1}{s}\left(e^{-\frac{Z}{2} \sqrt{s(s+4)}}\right)
$$

and $\theta=Z / 2$.

G(s) can be defined as the integral of Equation (53) as shown in Baumeister and Hamill [45]:

$$
G(s)=\int f(s) d Z=-\frac{2}{s \sqrt{s(s+4)}} e^{-\frac{Z \sqrt{s(s+4)}}{2}}
$$


The Laplace inverse of $G(s), G(\tau)$, can be written upon invoking the convolution property and the lag property given in (Mickley et al. [46]) Equation (50):

$$
G(\tau)=-2 \int_{Z}^{\tau} e^{-2\left(p-\frac{Z}{2}\right)} I_{0}\left(2 \sqrt{p^{2}-p Z}\right) d p
$$

Equation (56) is valid for $\tau>X$. The dimensionless temperature can be obtained by differentiating with respect to $Z$ (Equation (56)) and using the Leibnitz rule.

$$
u(Z, \tau)=2 e^{-Z}-2 \int_{Z}^{p} e^{-2 p} e^{Z} I_{0}\left(2 \sqrt{p^{2}-p Z}\right) d p+4 \tau \int_{Z}^{P} e^{-2\left(p-\frac{Z}{2}\right)} \frac{I_{1}\left(2 \sqrt{p^{2}-p Z}\right)}{\sqrt{p^{2}-p Z}} d p
$$

For conditions where $\tau=X$, the dimensionless temperature is given by:

$$
u=e^{-\frac{X}{2}}=e^{\frac{\tau}{2}}
$$

The binomial infinite series expansion was written for the power exponentiation in Equation (53) in Sharma [43]. By invocation of the convolution property the analytical solution may be obtained to varying degrees of accuracy. An approximate solution by truncation of the fourth- and higher-order terms in Equation (53) leads to the solution for transient temperature. Upon using the lag property as shown in Equation (54), the solution for transient temperature is given as follows in Equation (59):

$$
u \cong e^{-\frac{Z}{2}} I_{0}\left(\sqrt{\frac{Z \tau}{2}-\frac{3 Z^{2}}{4}}\right)
$$

The solution to the transient temperature with everything else such as the semi-infinite medium, boundary and time conditions used remaining the same, except that the Fourier model was used, can be written as follows in Equation (60):

$$
u=1-\operatorname{erf}\left(\frac{Z}{\sqrt{4 \tau}}\right)
$$

The solution to the transient temperature, with everything else such as the semi-infinite medium, boundary and time conditions used remaining the same, except that the damped wave conduction and relaxation model was used, can be written as follows in Equation (61):

$$
u=\frac{I_{0}\left(\frac{\tau^{2}-X^{2}}{4}\right)^{0.5}}{I_{0}\left(\frac{\tau}{2}\right)}
$$

Equation (61) is applicable for conditions where $\tau>X$. For conditions where $\tau<X$, the dimensionless temperature is given by:

$$
u=\frac{J_{0}\left(\frac{X^{2}-\tau^{2}}{4}\right)^{0.5}}{I_{0}\left(\frac{\tau}{2}\right)}
$$


For conditions where $\tau=X$, the dimensionless temperature is given by:

$$
u=e^{-\frac{X}{2}}=e^{-\frac{\tau}{2}}
$$

The predictions for transient concentration in a semi-infinite medium subject to the constant wall concentration (CWC) boundary condition from the (i) Fick model; (ii) damped wave conduction and relaxation model and; (iii) ballistic/acceleration model are plotted in Figure 2 side-by-side for the same set of parameters at $\tau=0.5$. The theoretical predictions from the ballistic model as given by Equation (59) were found to be closer in numerical value to the theoretical prediction from the Fick model as given by Equation (60) compared with that of the theoretical prediction from the damped wave conduction and relaxation model given by Equation (61). The transient concentration can be seen to be convex at short distances and changes to concave at later distances in the damped wave conduction and relaxation model. The transient concentration from the ballistic model is also convex at shorter distances and changes to concave later as a function of distance. The Fick parabolic model for transient concentration is concave as a function of distance. Equation (61) is valid in the open interval of $\tau>1.5$. For values of space and time when $\tau<1.5 \mathrm{Z}$ :

$$
u \cong e^{-\frac{Z}{2}} J_{0}\left(\sqrt{\frac{3 Z^{2}}{4}-\frac{Z \tau}{2}}\right)
$$

when $\tau=1.5 \mathrm{Z}$, the expression for transient temperature can be written as:

$$
u=e^{-\frac{Z}{2}}=e^{-\frac{\tau}{3}}
$$

The first zero of the Bessel function $J_{0}(y)$ occurs at $y=2.4048$. Thus, the penetration distance $Z_{\text {pen }}$ can be estimated for a given instant of time $\tau$ as follows:

$$
2.4048=\frac{3 Z_{\text {pen }}^{2}}{4}-\frac{Z_{\text {pen }} \tau}{2}
$$

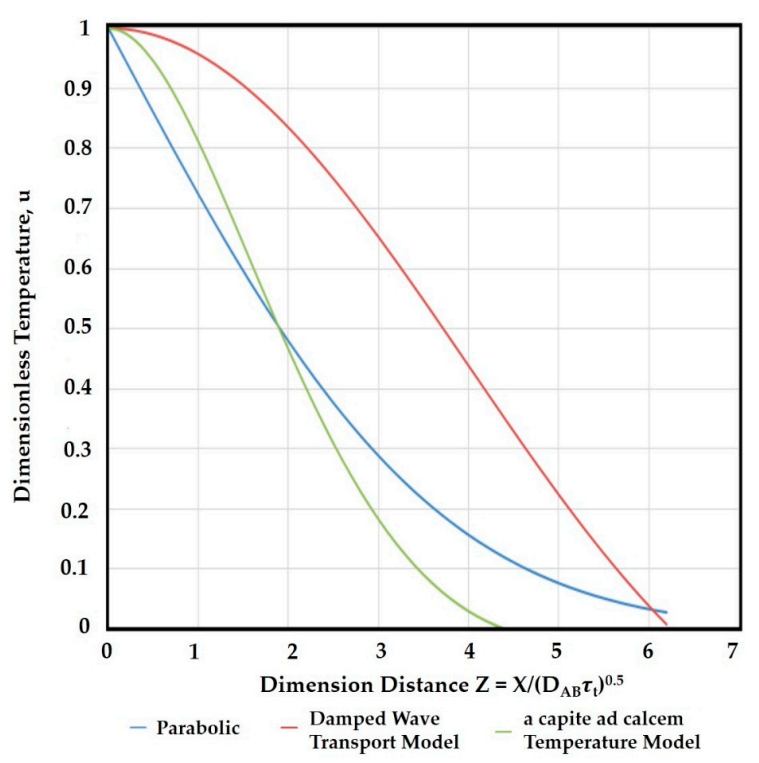

Figure 2. Comparison of transient concentration from Fick model, damped wave transport model and ballistic transport model. 


\section{Conclusions}

Fourier's law of heat conduction was derived at steady state from empirical observations. Equation (21) was proposed $[18,25,26]$ as an alternate to Fourier's law of heat conduction equation. This constitutive law was found to be in violation of the second law of thermodynamics under "certain conditions" $[1,35,36]$. In this study, the acceleration motion of a spinless electron was considered. The acceleration term was included. Eliminating the acceleration term between the equation of motion for the spinless electron and the heat flux formulation in free electron theory, an alternate form of heat conduction with contributing terms in temperature results, as given by Equation (19) and (20).

The velocity of electron was $95 \%$ of the velocity of heat. At steady state, for the spinless electron, Fourier's law of heat conduction is recovered from Equation (19) and (20). When the acceleration of the electron is taken as the time derivative of heat flux, Equation (21) results.

The entropy production term was considered for the law of heat conduction with contributing terms in temperature. It can be seen that the entropy production term will always be positive for real heat transfer events. For real heat transfer events, the heat flux to a point in the medium and the time rate of the change of temperature at that point in the medium can either both be positive or both be negative but can never, ever be one positive and one negative. As heat transmits to the point, the temperature will increase. In this case, both $q$ and accumulation in temperature will be positive. When heat is removed from the point, $q$ is negative and the time rate of temperature at that point will also be negative as the temperature will decrease.

A similar equation with contributing terms in concentration was derived for diffusion events using the Stokes-Einstein formulation. Soft materials, oligonucleotides, were considered. Transport parameters are a strong function of material type.

Model solutions for the diffusion of mass in semi-infinite medium subject to a step change in concentration were obtained using the method of Laplace transforms. The governing equation used included the a capite ad calcem in the concentration equation. The model solutions from the a capite ad calcem in concentration, the Maxwell-Cattaneo-Vernotte model and the Fick model were compared side by side and it is shown in Figure 2.

It was found that the a capite ad calcem model was closer in predictions to the Fick model. Both with contributing terms in concentration and the damped wave model exhibited convex curvature at short distances. This also means there is bounded flux at the surface. The Fick model has infinite slope at short times at the surface. The convex-concave transition was found to be sooner in the case of the a capite ad calcem in concentration model. This happened at a dimensionless distance of 2.0 at $\tau=4.0$. The convex curvature in both the damped wave and a capite ad calcem models can be used to remove the "blow-up" found in the Fick model for short time applications.

The relaxation time can be estimated from the diffusion coefficient, molecular weight and temperature. The thermal relaxation time can be estimated from the thermal conductivity of the material, pressure and heat capacity. The thermal relaxation time varies inversely with pressure and the mass relaxation time increases with an increase in molecular weight. For materials where the diffusion coefficient varies linearly with temperature, the mass relaxation times can be expected to be independent of the temperature. Low gravity orbital flight experiments are underway to evaluate this further.

Acknowledgments: At the ouset, the dynamism and timeliness shown by Elsevier, Amsterdam, Netherlands, for bringing out Damped Wave Transport and Relaxation in 2005 is appreciated. Thanks to the encouragement from four sources in three different continents; (i) R. Sethuraman, Vice Chancellor at SASTRA University, Thanjavur, India, for the Cash Prize for authoring an international book received in 2005; (ii) President of Prairie View A \& M University, Prairie View, TX, George Wright's Merit Pay received in 2011 based upon Performance Review and; (iii) Honoroary Fellowship Activities, Australian Institute of High Energetic Materials, Monash University, Melbourne, Australia, and (iv) formerly President, RTI Intl., RTP, NC, Victoria Franchetti Haynes for selecting me to serve on the "fast-track" at Monsanto Plastics Technology, Indian Orchad, MA, on an August day in 1991. Vote of thanks to Harris County Public Library, CyFair Location, Houston, TX, for unrestricted use of desktop computer with Intel processors with $2.7 \mathrm{GHz}$ speed, 2 GB memory and 32 bit operating systems where the graphs in the work were prepared in MS Windows Excel 2013 software. Last but not the least, thanks to all those who 
called my attention to transient applications where the model capability from use of Fourier's representation of transient temperature is not sufficient to describe the real events.

Conflicts of Interest: The author declare no conflict of interest.

\section{Abbreviations}

$A_{\mathrm{N}}$

A

$C_{\mathrm{A}}$

$C_{B}$

$C_{\mathrm{p}}$

$C_{\mathrm{v}}$

$D_{\mathrm{AB}}$

$\operatorname{erf}(z)$

f

$k$

$\mathrm{k}_{\mathrm{B}}$

$H$

$J^{\prime \prime}$

$J_{\mathrm{p}}(x)$

$I_{\mathrm{p}}(x)$

$t$

T

m

N

$q_{\mathrm{z}}$

$n$

$\mathrm{R}$

$R_{0}$

$S$

u

$u(\mathrm{~s})$

$v_{\mathrm{e}}$

$v_{\mathrm{A}}$

$v_{\mathrm{y}}$

$v_{\mathrm{m}}$

z

Z

$v_{\mathrm{h}}$

$Z_{\text {pen }}$

\section{Greek}

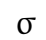

$\alpha$

$\mathrm{t}$
Avagadro Number $\left(6.023 \times 10^{23}\right.$ molecules $/$ mole $)$

cross-sectional area across which transport occurs $\left(\mathrm{m}^{2}\right)$

concentration of species A $\left(\mathrm{mol} / \mathrm{m}^{3}\right)$

concentration of species B $\left(\mathrm{mol} / \mathrm{m}^{3}\right)$

heat capacity of material at constant pressure $(\mathrm{J} / \mathrm{mole} / \mathrm{K})$

heat capacity of material at constant volume $(\mathrm{J} / \mathrm{mole} / \mathrm{K})$

binary diffusion coefficient of species $\mathrm{A}$ in $\mathrm{B}\left(\mathrm{m}^{2} / \mathrm{s}\right)$

error function of z. $\operatorname{erf}(z)=\frac{2}{\sqrt{\pi}} \int_{0}^{z} e^{-z^{2} d z}$

molecular drag coefficient $(\mathrm{kg} / \mathrm{s} /$ molecule)

thermal conductivity of the material $(\mathrm{W} / \mathrm{m} / \mathrm{K})$

Boltzmann Constant (J/molecule/K)

enthalpy $(\mathrm{J} / \mathrm{mole})$

area averaged molar flux (mole $\left./ \mathrm{m}^{2} / \mathrm{s}\right)$

Bessel function of the $\mathrm{p}^{\text {th }}$ order and first kind

modified Bessel function of the $\mathrm{p}^{\text {th }}$ order and first kind

time (s)

Temperature $\left({ }^{\circ} \mathrm{K}\right)$

mass of the molecule $(\mathrm{kg})$

molecular weight of oligonucleotide $(\mathrm{kg} / \mathrm{mole})$

heat flux (area averaged) $\left(\mathrm{W} / \mathrm{m}^{2}\right)$

electron density (\# of electrons $/ \mathrm{m}^{3}$ )

universal molar gas constant $(\mathrm{J} / \mathrm{mole} / \mathrm{K})$

radius of solute molecule $(\mathrm{m})$

entropy $(\mathrm{J} / \mathrm{mole} / \mathrm{K})$

dimensionless concentration, $u=\left(\frac{C_{A}-C_{0}}{C_{A s}-C_{0}}\right)$

temperature in Laplace domain

velocity of electron $(\mathrm{m} / \mathrm{s})$

velocity of solute molecule $(\mathrm{m} / \mathrm{s})$

velocity of fluid in y cartesian direction $(\mathrm{m} / \mathrm{s})$

velocity of mass $(\mathrm{m} / \mathrm{s})$

$\mathrm{z}$ Cartesian distance $(\mathrm{m}) \mathrm{Z}=\left(\frac{x}{\sqrt{D_{A B} \tau_{m r}}}\right)$

dimensionless distance

velocity of heat $(\mathrm{m} / \mathrm{s})$

dimensionless penetration distance

Entropy production term $\left(\mathrm{W} / \mathrm{m}^{3} / \mathrm{K}\right)$

thermal diffusivity of material $\left(\mathrm{m}^{2} / \mathrm{s}\right)$

collision time of the electron and obstacle (seconds) 
$\mu$

Dimensionless time in governing equation $\tau=\left(\frac{t}{\tau_{r}}\right)$

relaxation time (heat) of material (s)

relaxtion time (mass) of material (s)

density of material $\left(\mathrm{kg} / \mathrm{m}^{3}\right)$

tangential shear stress $\left(\mathrm{N} / \mathrm{m}^{2}\right)$

relaxtion time (momentum) (s)

chemical potential $(\mathrm{J} /$ molecule)

viscosity $(\mathrm{kg} / \mathrm{m} / \mathrm{s})$

retardation time (s)

mole fraction of species A

\section{References}

1. Jou, D.; Casas-Vazquez, J.; Lebon, G. Extended Irreversible Thermodynamics, 3rd ed.; Springer Verlag: Berlin, Germany, 2001.

2. Onsager, L. Reciprocal Relations in Irreversible Processes. Phys. Rev. 1931, 37, 405-426. [CrossRef]

3. Fourier, J.B. Theorieanalytique de la Chaleur; Freeman, A., Translator; Dover Publications: New York, NY, USA, 1955.

4. Fick, A. Annalen der Physik; Wiley: Leipzig, Germany, 1855; Volume 170, p. 59.

5. Ohm, G.S. GrundlinienzueinerzweckmäßigenBehandlung dereometriealshöherenBildungsmittelsanvor bereitendenLehranstalten/entworfen (Guidelines for an Appropriatetreatment of Geometry in Higher Education at Preparatory Institutes/Notes); Palm und Enke: Erlangen, Germany, 1817.

6. Newton, I. Philosophiae Naturalis Principia Mathematica; Edmund Halley: London, UK, 1687.

7. Sharma, K.R. Damped Wave Conduction and Relaxation in Cylindrical and Spherical Coordinates. J. Thermophys. Heat Transf. 2007, 21, 688-693. [CrossRef]

8. Sharma, K.R. On the Solution of Damped Wave Conduction and Relaxation Equation in a Semi-Infinite Medium Subject to Constant Wall Flux. Int. J. Heat Mass Transf. 2008, 51, 25-26, 6024-6031. [CrossRef]

9. Sharma, K.R. A Fourth Mode of Heat Transfer called Damped Wave Conduction. In Proceedings of the 42nd Annual Convention of Chemists Meeting, Santiniketan, India, 10 February 2006.

10. Sharma, K.R. Damped Wave Transport and Relaxation; Elsevier: Amsterdam, The Netherlands, 2005.

11. Renganathan, K. Correlation of Heat Transfer with Pressure Fluctuations in Gas-Solid Fluidized Beds to Immersed Surfaces. Ph.D. Dissertation, West Virginia University, Morgantown, WV, USA, 1990.

12. Sharma, K.R. Storage Coefficient of Substrate in a $2 \mathrm{GHz}$ Microprocessor. In Proceedings of the 225th ACS National Meeting, New Orleans, LA, USA, 23-27 March 2003.

13. Sharma, K.R. Bioinformatics:Sequence Alignment and Markov Models; McGraw Hill: NewYork, NY, USA, 2009.

14. Sharma, K.R. Errors in Gel Acrylamide Electrophoresis Due to Effect of Charge. In Proceedings of the 231st ACS National Meeting, Atlanta, GA, USA, 26-30 March 2006.

15. Sharma, K.R. Polymer Thermodynamics: Blends, Copolymers and Reversible Polymerization; CRC Press/Taylor \& Francis Group: Baco Raton, FL, USA, 2012.

16. Sharma, K.R. On Analytical Solutions during Damped Wave Conduction and Relaxation in a Finite Slab Subject to the Convective Boundary Condition. Int. J. Thermophys. 2010, 31, 430-443. [CrossRef]

17. Sharma, K.R. Transport Phenomena in Biomedical Engineering: Artificial Organ Design and Development and Tissue Design; McGraw Hill Professional: New York, NY, USA, 2010.

18. Sharma, K.R. On Damped Wave Diffusion of Oxygen in Islets of Langerhans: Part I Comparison of Parabolic and Hyperbolic Models in a Finite Slab. In Proceedings of the 102nd AIChE Annual Meeting, Salt Lake City, UT, USA, 12 November 2010.

19. Sharma, K.R. Nanostructuring Operations in Nanoscale Science and Engineering; McGraw Hill Professional: New York, NY, USA, 2010.

20. Sharma, K.R. Solution Methods and Applications for Generalized Fick's Law of Diffusion. In 43rd Annual Conventtion of Chemists; Indian Chemical Society: Aurangabad, Gujarat, India, 2006. 
21. Sharma, K.R. Comparison of Solutions from Parabolic and Hyperbolic Models for Transient Heat Conduction in Semi-Infinite Medium. Int. J. Thermophys. 2009, 30, 1671-1687. [CrossRef]

22. Sharma, K.R.; Turton, R. Mesoscopic Approach to Correlate Surface Heat Transfer Coefficients with Pressure Fluctuations in Dense Gas-Solid Fluidized Beds. Powder Technol. 1998, 99, 109-118. [CrossRef]

23. Landau, L.; Liftshitz, E.M. Fluid Mechanics; Publisher: Pergamon, UK, 1987.

24. Casimir, H.B.G. Note on the Conduction of Heat in Crystals. Physica 1938, 5, 495-500. [CrossRef]

25. Cattaneo, C. A Form of Heat Conduction which Eliminates the Paradox of Instantaneous Propagation. Comptes Rendus 1958, 247, 431-433.

26. Vernotte, P. Les Paradoxes de la Theorie Continue de l'equation de la Chaleur. C. R. Acad. Sci. Paris 1958, 246, 3154-3155.

27. Maxwell, J.C. On the Dynamical Theory of Gases. Phil. Trans. Roy. Soc. 1867, 157, 49-88. [CrossRef]

28. Joseph, D.D.; Preziosi, L. Heat Waves. Rev. Mod. Phys. 1989, 61, 41-73. [CrossRef]

29. Ozisik, M.N.; Tzou, D.Y. On the Wave Theory of Heat Conduction. ASME J. Heat Transf. 1994, 116, 526-535. [CrossRef]

30. Sharma, K.R. Continuous Process, Dynamics, Stability, Automation and Control, Nova Science; Nova Science: Happauge, NY, USA, 2015.

31. Sharma, K.R. Analytical Solution of Damped Wave Conduction and Relaxation in a Finite Sphere and Cylinder. J. Thermophys. Heat Transf. 2008, 22, 783-786. [CrossRef]

32. Sharma, K.R. Lecture Notes in Computational Molecular Biology; SASTRA Press: Kumbakonam, India, 2004.

33. Sharma, K.R. Transport Phenomena in Biomedical Engineeirng: Artifical Organ Design and Development and Tissue Design; McGraw Hill Professionl: New York, NY, USA, 2010.

34. Taitel, Y. On the Parabolic, Hyperbolic and Discrete Formulation of Heat Conduction Equation. Int. J. Heat Mass Transf. 1972, 15, 369-371. [CrossRef]

35. Bai, C.; Lavine, A.S. On Hyperbolic Heat Conduction and Second Law of Thermodynamics. J. Heat Transf. 1995, 117, 256-263. [CrossRef]

36. Zanchini, E. Hyperbolic Heat Conduction Theories and non-Decreasing Entropy. Phy. Rev. B 1999, 60, 991-997. [CrossRef]

37. Lorentz, H.A. La Théorieelectromagnétique de Maxwell et sonapplication aux corps mouvants, Archives néerlandaises des sciences exactes et naturelles. Intern. Arch. 1892, 25, 363-552. (In French).

38. Drude, P. ZurElektronentheorie der metalle. Ann. Physik 1900, 306, 566-613. (In German). [CrossRef]

39. Boltzmann, L. Über die Natur der Gasmoleküle (On the nature of gas molecules). WienerBerichte 1876, 74, 553-560.

40. Sharma, K.R. On the Derivation of an Expression for Relaxation Time from Stokes-Einstein Relation. In Proceedings of the 233rd ACS National Meeting, Chicago, IL, USA, 25-29 March 2007; American Chemical Society: Washington, DC, USA, 2007.

41. Sharma, K.R. Manifestation of Acceleration during Transient Heat Conduction. J. Thermophys. Heat Transf. 2006, 20, 799-808. [CrossRef]

42. Bird, R.B.; Stewart, W.E.; Lightfoot, E.N. Transport Phenomena, 2nd ed.; John Wiley \& Sons: New York, NY, USA, 2007.

43. Sharma, K.R. Microarray Analysis: Biochips and Eradication of All Diseases; Momentum Press: New York, NY, USA, 2015.

44. Sharma, K.R. Principles of Mass Transfer; Prentice Hall of India: New Delhi, India, 2007.

45. Baumeister, K.J.; Hamill, T.D. Hyperbolic Heat Conduction Equation-A Solution for the Semi-Infinite Body Problem. ASME J. Heat Transf. 1971, 93, 126-128. [CrossRef]

46. Mickley, H.S.; Sherwood, T.K.; Reid, C.E. Applied Mathematics in Chemical Engineering; McGraw Hill: New York, NY, USA, 1957.

(C) 2015 by the author; licensee MDPI, Basel, Switzerland. This article is an open access article distributed under the terms and conditions of the Creative Commons by Attribution (CC-BY) license (http://creativecommons.org/licenses/by/4.0/). 